\title{
Bilateral Finger Jerks as a Useful Sign for Diagnosis of Cervical Compressive Myelopathy
}

\author{
Naoki Kasahata
}

\begin{abstract}
Background: Bilateral finger jerks are very frequently observed when we examine cervical compressive myelopathy patients. It is very important to determine what sign or symptom is useful for the early diagnosis of cervical compressive myelopathy.
\end{abstract}

Methods: We studied 24 patients with cervical compressive myelopathy. We studied neurological signs and symptoms (bilateral finger jerks, the attitude of deep tendon reflexes, patients' complaints especially tingling sensations of hands, Hoffmann's signs) and compared with level diagnoses made by T2-weighted MRI.

Results: Eleven patients showed bilateral finger jerks: among these 11 patients, seven patients showed hyperreflexia of biceps and brachioradial reflexes and four patients showed hyporeflexia (inverted radial reflex). All patients with bilateral finger jerks showed lesions at the C5-6 or C4-5 intervertebral levels (C7 or C6 spinal cord levels) on MRI. Among four patients who showed bilateral finger jerks with hyporeflexia (inverted radial reflexes), two patients showed lesions at C5-6 intervertebral level (C7 spinal cord level). Nine patients showed generalized hyperreflexia. 14 patients developed tingling sensations of the hands: among these 14 patients, five patients were affected unilaterally and nine patients were affected bilaterally. Among these 14 patients with tingling sensations of the hands, seven had lesions at the C4-5 intervertebral levels and six had C5-6 intervertebral levels. Sensory levels were observed in five patients. Hoffmann's signs were not observed in any patients.

Manuscript accepted for publication April 19, 2011

Department of Neurology, Makita General Hospital, Tokyo, and, Division of Neurology, Department of Internal Medicine, Tokyo Metropolitan Ohtsuka Hospital, Tokyo, Japan Correspondence: Division of Neurology, Department of Internal Medicine, Tokyo Metropolitan Ohtsuka Hospital, Minamiohtsuka 2-8-1, Toshima-ku, Tokyo 170-8476, Japan.

Email:n_kasa-o@ohtsuka-hospital.toshima.tokyo.jp

doi: $10.4021 / \mathrm{jnr} 15 \mathrm{w}$
Therefore, 23 of 24 (96\%) cervical compressive myelopathy patients showed bilateral finger jerks or generalized hyperreflexia or complained tingling sensations of hands.

Conclusions: We must conclude that deep tendon reflex examination especially the observation of bilateral finger jerks or generalized hyperreflexia and history taking of tingling sensations of hands are useful for the screening of cervical compressive myelopathy.

Keywords: Bilateral finger jerks; Cervical compressive myelopathy; Generalized hyperreflexia; Tingling sensations of hands; Segmental sign

\section{Introduction}

Bilateral finger jerks are very frequently observed when we examine cervical compressive myelopathy patients. Early diagnosis of cervical compressive myelopathy is essential for relief of patients' symptoms and some of them may need surgical treatment. Therefore, it is very important to determine what sign or symptom is useful for the early diagnosis of cervical compressive myelopathy.

Considerable research has been conducted to find useful clinical signs or syndromes for early or accurate anatomical diagnosis of cervical compressive myelopathy. The average accuracy of neurologic level diagnosis has been reported to be $70 \%$ or more, while diagnosis of the level by sensory disturbance area showed the highest accuracy [1]. Among the neurological tests, patient-perceived location of numbness in the hand was the most useful for establishing the affected level [2]. Patients with protruded discs at C5-6 described dysesthesia in the thumb and index finger with deep tendon reflexes in the biceps and brachioradialis being diminished. Patients with protruded discs at the C6-7 level described dysesthesia in the middle finger and their deep tendon reflexes in the triceps were diminished. Patients with C3-4 protruded discs did not complain of dysesthesia in any digits and their deep tendon reflexes were exaggerated in all muscles in the upper extremities [3].

Cervical myelopathy patients with false localizing tho- 
racic sensory levels presented with progressive weakness and hyperreflexia involving the lower extremities and distinct thoracic sensory levels ranging from T-4 to T-10 [4]. Patients who presented with clearly defined thoracic sensory levels were found to have cervical spinal cord compression [5]. Patients with midline cervical disc protrusion at the C3-4 intervertebral level showed unusual clinical signs; numbness in the finger tips and palms, clumsiness of the hands, and a tightening sensation at the midthoracic level [6]. Cervical angina, defined as chest pain that resembles true cardiac angina but originates from cervical discopathy, most commonly results from compression of C7 [7]. The presence of a prominent pectoralis jerk was seen only in patients with spinal cord compression at the $\mathrm{C} 2-3$ and/or C3-4 levels [8]. A wide-based spastic gait is the classical presentation of patient with cervical spondylotic myelopathy [9].

The presence of a positive Hoffmann's sign in asymptomatic patients strongly suggests underlying cervical pathology [10]. A positive dynamic Hoffmann's sign was reported as a useful clinical sign for early diagnosis of cervical spondylotic myelopathy [11].

While considerable research in cervical compressive myelopathy has been reported, the usefulness of bilateral finger jerks for diagnosis of cervical compressive myelopathy remains largely unknown. Finger jerk is elicited by biceps brachii reflex or brachioradial reflex. If finger jerk is associated with absent biceps brachii reflex or brachioradial reflex, it is interpreted as an indication of C5 or C6 segmental spinal cord lesion sign (inverted radial reflex). If finger jerk is associated with increased biceps brachii reflex or brachioradial reflex, it is a sign associated with hyperreflexia. Therefore, bilateral finger jerks with hyperreflexia are interpreted as an indication of a corticospinal tract lesion more rostral than the $\mathrm{C} 6$ (or $\mathrm{C} 5$ ) segment of spinal cord. It is well known that the finger flexors are mostly innervated from the C6, C7, C8 and T-1 segment, so that it stands to reason for the finger jerk with a cord lesion at higher levels.

This article investigated the utility of bilateral finger jerks and other neurological signs for diagnosis of cervical compressive myelopathy. This paper retrospectively evaluated the neurological characteristics of 24 cervical compressive myelopathy patients.

\section{Materials and Methods}

We defined cervical compressive myelopathy as cervical myelopathy owing to extramedullary compressive lesions such as cervical spondylosis, herniation of intervertebral disk, ossification of posterior longitudinal ligament, ossification of ligamentum flavum, and vertebral sliding. Patients $(\mathrm{n}=24)$ were cervical compressive myelopathy patients recruited from the Department of Neurology of the Makita General Hospital between April 2004 and April 2006. All pa- tients were initially outpatients and some were admitted to the hospital during the course of the investigation. Some of them underwent surgical treatment. All patients complained of symptoms compatible with cervical compressive myelopathy.

All patients were diagnosed by magnetic resonance imaging (MRI). And all showed symptoms that could be explained by the lesions detected on MRI. We studied the levels of lesions, bilateral finger jerks, the state of deep tendon reflexes, sensory levels, Hoffmann's signs and the characteristics of their symptoms. Same examiner evaluated all patients.

We defined finger jerk as reflex flexion of fingers on tapping the biceps tendon or eliciting the brachioradial reflex. We defined Hoffmann's sign as reflex flexion of the thumb when the middle finger flicked from the palmar surface.

\section{Results}

\section{Case reports}

\section{Patient 1}

A 72-year-old woman developed a tingling sensation of her left hand especially in the morning. Neurological examination showed generalized hyperreflexia with bilateral finger jerks at biceps and brachioradial reflexes, C7 sensory level, and gait disturbance. Her left hand was unable to perform rapid alternative movement well. She showed a mild scissors gate. She was unable to perform tandem gait. MRI demonstrated C4-5 and C5-6 myelopathy with a boomerang-like change (Fig. 1A, 2A, B). Thereafter, she developed weakness of the left hand, gait disturbance and dizziness. She became able to use a cane. However, use of a cervical collar improved her symptoms, and sensory level moved slightly rostral in the direction: at the first examination, it had been between thumbs and index fingers. After application of a cervical collar, the sensory level moved to the radial sides of the wrist.

\section{Patient 2}

A 64-year-old man developed tingling sensation of the bilateral hands, right shoulder pain and chest pain persisting for several seconds at night (approximately 2:00 A.M.). He developed right side dominant dull pain that felt like electric shock from his forearms to hands, when he was in a prone position especially in daytime. He did not complain of urinary disturbance, disturbance of defecation or impotence. One year earlier, Orthopedist diagnosed him as cervical spondylosis. Neurological examination showed hyperreflexia of bilateral biceps reflex, and areflexia of bilateral triceps. Other deep tendon reflexes were normal. There was absence 

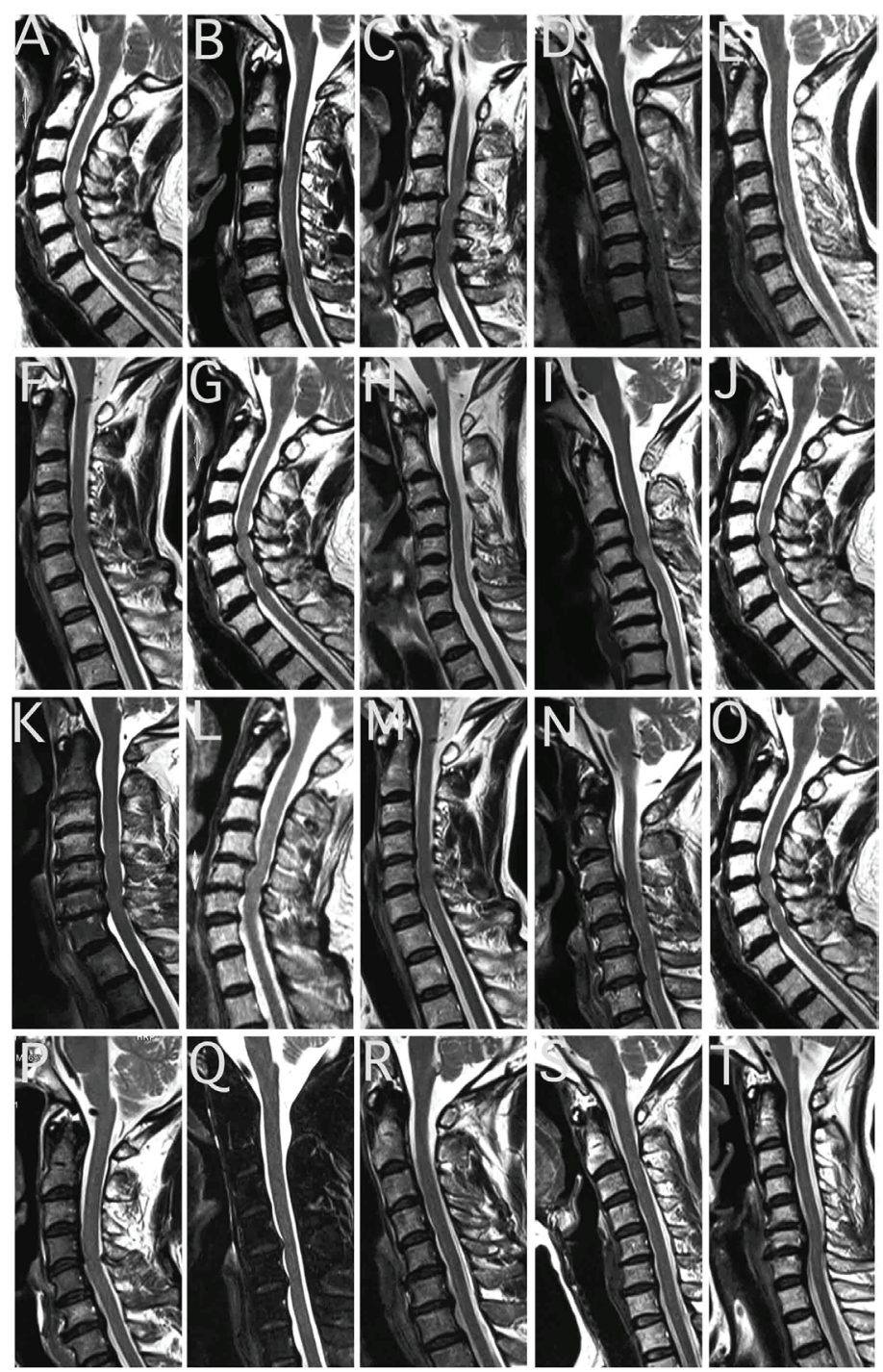

Figure 1. Sagittal T2-weighted magnetic resonance imaging (TR 500 TE 24). (A) Spinal cord compressions owing to C4-5, C5-6 intervertebral disk hernias were observed in Patient 1. (B) Spinal cord compressions owing to C6-7 (and mild C3-4) intervertebral disk hernias were observed in Patient 2. C3 vertebral level spinal cord showed a mild high intensity area and myelomalacia was suspected. (C) C3 vertebra was sliding posterior. C3-4, C4-5 (and mild C5-6) intervertebral level spinal cord compressions and spinal canal stenosis were observed in Patent 3. (D) Spinal cord compression owing to C5-6 intervertebral disk bulging and spinal canal stenosis were observed in Patient 4. (E) Spinal cord compression owing to C5-6 intervertebral level lesion was observed in Patient 5. (F) Spinal cord compression owing to C4-5 intervertebral disk hernia was observed in Patient 6. (G) Spinal cord compressions owing to C4-5 and C5-6 intervertebral disk bulging with osteophyte formations were observed in Patient 7. (H) Spinal cord compression owing to C3-4 intervertebral kyphosis deformity was observed in Patient 8. (I) Spinal cord compression owing to C4-5 intervertebral level lesion and a T2 high intensity area in C4 vertebral level spinal cord were observed in Patient 9. (J) Spinal cord compressions and deformity owing to C5-6 (and mild C4-5) intervertebral level lesions were observed in Patient 10. (K) Severe spinal cord compressions owing to C6-7 (mild C4-5 and C5-6) intervertebral disk bulging and osteophyte formations were observed in Patient 11. (L) Spinal cord compressions owing to C4-5 (and mild C5-6) intervertebral level lesions were observed in Patient 13. (M) Spinal cord compression owing to C4-5 intervetebral disk hernia was observed in Patient 17. (N) Spinal cord compression and mild deformity owing to C5-6 intervertebral disk hernia and hypertrophy of the posterior longitudinal ligament lesion were observed in Patient 18. (O) Spinal cord compressions owing to C5-6 (mild C4-5 and C6-7) intervertebral disk bulging were observed in Patient 19. (P) Spinal cord compression owing to C4-5 intervertebral disk bulging was observed in Patient 20. (Q) Spinal cord compressions owing to C4-5 and C5-6 intervertebral level osteophyte formations were observed in Patient 21. (R) Right side dominant spinal cord compressions owing to C4-5 and C5-6 intervertebral disk hernias were observed in Patient 22. (S) Spinal cord compression owing to C5-6 intervertebral disk bulging was observed in Patient 23. (T) Spinal cord compression owing to osteophyte formation of C6 vertebra and hypertrophy of posterior longitudinal ligament was observed in Patient 24. 

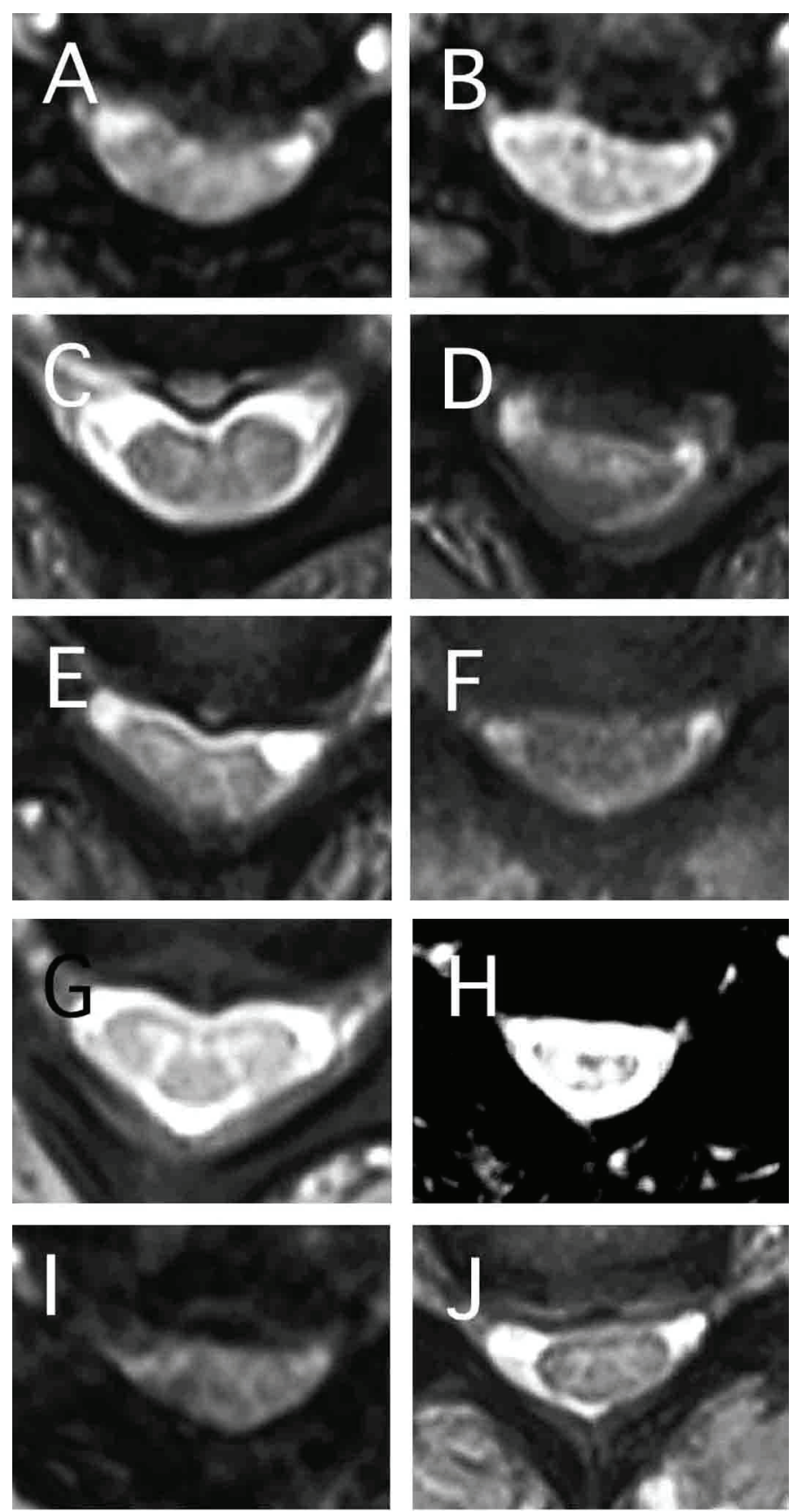

Figure 2. Axial T2-weighted magnetic resonance imaging (TR 500 TE 24). (A) C4-5 intervertebral level section of Patient 1. Spinal cord compression and boomerang-like change owing to C4-5 intervertebral disk hernia were observed. (B) C5-6 intervertebral level section of Patient 1. Spinal cord compression and boomerang-like change owing to C5-6 intervertebral disk hernia were observed. (C) C6-7 intervertebral level section of Patient 2. Spinal cord compression and mild boomerang-like change owing to C6-7 intervertebral disk hernia were observed. (D) C3-4 intervertebral level section of Patient 3. C3-4 intervertebral level spinal cord compression and spinal canal stenosis owing to C3 vertebral sliding were observed. (E) C4-5 intervertebral level section of Patient 3. C4-5 intervertebral level spinal cord compression and spinal canal stenosis owing to C3 vertebral sliding were observed. (F) C5-6 intervertebral level section of Patient 4. Spinal cord compression owing to C5-6 intervertebral disk bulging was observed. (G) C4-5 intervertebral level section of Patient 6. Spinal cord compression owing to C4-5 intervertebral disk hernia was observed. (H) C4-5 intervertebral level section of Patient 9. Spinal cord compression owing to C4-5 intervertebral level lesion was observed. (I) C4-5 intervertebral level section of Patient 13. Spinal cord compression and boomerang-like change owing to C4-5 intervertebral level lesion were observed. (J) C5-6 intervertebral level section of Patient 18. Spinal cord compression and mild deformity owing to C5-6 intervertebral disk hernia and hypertrophy of the posterior longitudinal ligament lesion were observed. 
Table 1

\begin{tabular}{|c|c|c|c|c|c|}
\hline Patient & Age, Gender & Vertebral level & DTRs & Sensory levels & Sensory and other symptoms \\
\hline 1 & $72, \mathrm{~F}$ & $\mathrm{C} 4-5, \mathrm{C} 5-6, \mathrm{C} 6-7$ & hyper & C7 & tingling sensation of left hand \\
\hline 2 & $64, \mathrm{M}$ & $\mathrm{C} 6-7,(\mathrm{C} 3-4)$ & variable & - & tingling of both hands, chest pain \\
\hline 3 & $71, \mathrm{M}$ & $\mathrm{C} 3-4, \mathrm{C} 4-5,(\mathrm{C} 5-6)$ & hyper & - & gait dis. \\
\hline 4 & $62, \mathrm{~F}$ & $\mathrm{C} 5-6$ & variable & - & head hyperhidro., body hypohidro \\
\hline 5 & $65, \mathrm{~F}$ & $\mathrm{C} 5-6$ & hyper & - & tingling sensations of both feet \\
\hline 6 & $62, \mathrm{~F}$ & $\mathrm{C} 4-5$ & variable & - & tingling of all extremities \\
\hline 7 & $63, \mathrm{M}$ & $\mathrm{C} 4-5, \mathrm{C} 5-6$ & $\mathrm{a}$ & - & tingling sensation of right hand \\
\hline 8 & $82, \mathrm{~F}$ & $\mathrm{C} 3-4$ & hyper & - & tingling, weakness of both hands \\
\hline 9 & $76, \mathrm{~F}$ & $\mathrm{C} 4-5$ & $\mathrm{a}$ & C7-8 & tingling of both hands, gait dis. \\
\hline 10 & $46, \mathrm{M}$ & $\mathrm{C} 5-6(\mathrm{C} 4-5)$ & $\mathrm{a}$ & - & gait dis., anuresis \\
\hline 11 & $74, \mathrm{M}$ & $\mathrm{C} 6-7$ (C4-5, C5-6) & variable & $\mathrm{C} 4-5$ & tingling sensations of both hands \\
\hline 12 & $53, \mathrm{~F}$ & $\mathrm{C} 5-6$ & hypo & - & tingling, numbness of both hands \\
\hline 13 & $64, \mathrm{~F}$ & $\mathrm{C} 4-5(\mathrm{C} 5-6)$ & hypo & $\mathrm{C} 6$ & weakness of left hand \\
\hline 14 & $71, \mathrm{~F}$ & C5-6 & hypo & C5-6 & tingling of lower extremities \\
\hline 15 & $76, \mathrm{~F}$ & $\mathrm{C} 4-5$ & hyper & - & tingling of all extremities \\
\hline 16 & $74, \mathrm{M}$ & $\mathrm{C} 4-5$ & hyper & - & gait dis. \\
\hline 17 & $65, \mathrm{~F}$ & $\mathrm{C} 4-5$ & hyper & - & tingling sensation of right hand \\
\hline 18 & $62, \mathrm{M}$ & C5-6 & normo & - & tingling of left hand, gait dis. \\
\hline 19 & $81, \mathrm{~F}$ & C5-6, (C4-5 (C6-7)) & hyper & - & chest discomfort \\
\hline 20 & $69, \mathrm{M}$ & $\mathrm{C} 4-5$ & hypo & - & unsteadiness \\
\hline 21 & $38, \mathrm{M}$ & $\mathrm{C} 4-5, \mathrm{C} 5-6$ & hyper & - & stiffness of left forearm \\
\hline 22 & $58, \mathrm{M}$ & $\mathrm{C} 4-5, \mathrm{C} 5-6$ & $\mathrm{a}$ & - & tingling sensation of right hand \\
\hline 23 & $53, \mathrm{~F}$ & $\mathrm{C} 5-6$ & variable & - & tingling sensations of both hands \\
\hline 24 & $58, \mathrm{~F}$ & $\mathrm{C} 6$ & $\mathrm{a}$ & - & tingling, clumsiness of both hands \\
\hline
\end{tabular}

of Hoffmann's sign. MRI demonstrated C6-7 myelopathy (and mild C3-4 myelopathy) and mild boomerang-like deformity of the cervical cord (Fig. 1B, 2C).

\section{Patient 4}

A 62-year-old woman developed hyperhidrosis of the head and hypohidrosis of other body areas. Neurological exami- nation showed generalized hyperreflexia with bilateral finger jerks of the biceps and brachioradial reflexes, there was absence of Hoffmann's sign and absence of apparent sensory level. MRI demonstrated C5-6 myelopathy (Fig. 1D, 2F).

\section{Patient 6}

A 62-year-old woman developed tingling sensations of all 
Table 2

\begin{tabular}{|c|c|c|c|c|c|c|}
\hline Patient & Vertebral level & Biceps & Brachioradial & Triceps & Finger jerks & Long tract sign \\
\hline 1 & $C 4-5,5-6$ & +++ & +++ & +++ & + & gait dis., sensory level \\
\hline 2 & C6-7 (3-4) & +++ & ++ & - & - & chest pain, gait dis. \\
\hline 3 & $\mathrm{C} 3-4,4-5(5-6)$ & +++ & +++ & +++ & + & gait dis. \\
\hline 4 & $\mathrm{C} 5-6$ & +++ & +++ & +++ & + & hypohidrosis of body \\
\hline 5 & $\mathrm{C} 5-6$ & +++ & +++ & +++ & - & tingling sensations of $\mathrm{L} / \mathrm{E}$ \\
\hline 6 & $\mathrm{C} 4-5$ & $++/+++$ & $++/+++$ & $++/+++$ & + & $\begin{array}{l}\text { weakness, sensory disturbance } \\
\text { of } \mathrm{L} / \mathrm{E}\end{array}$ \\
\hline 7 & $C 4-5,5-6$ & - & - & $-/++$ & - & hyperreflexia of L/E \\
\hline 8 & $\mathrm{C} 3-4$ & +++ & +++ & ++ & - & hyperreflexia of L/E \\
\hline 9 & $\mathrm{C} 4-5$ & - & - & ++ & - & gait dis. \\
\hline 10 & $\mathrm{C} 4-5$ & - & - & ++ & + & gait dis., anuresis, impotence \\
\hline 11 & C6-7 (4-5, 5-6) & + & - & ++ & - & hyperreflexia of L/E \\
\hline 12 & $\mathrm{C} 5-6$ & + & $-/+$ & + & + & $\begin{array}{l}\text { pain of both toes, increased right } \\
\text { PTR }\end{array}$ \\
\hline 13 & $\mathrm{C} 4-5(5-6)$ & ++ & - & ++ & + & hyperreflexia of L/E \\
\hline 14 & $\mathrm{C} 5-6$ & $+/+++$ & ++ & + & + & tingling sensation of right $\mathrm{L} / \mathrm{E}$ \\
\hline 15 & $\mathrm{C} 4-5$ & +++ & +++ & ++ & - & tingling and hyperreflexia of $\mathrm{L} / \mathrm{E}$ \\
\hline 16 & $\mathrm{C} 4-5$ & +++ & +++ & +++ & - & $\begin{array}{l}\text { spasticity, hyperreflexia of } \mathrm{L} / \mathrm{E} \text {, } \\
\text { gait dis. }\end{array}$ \\
\hline 17 & $\mathrm{C} 4-5$ & +++ & +++ & ++ & + & (finger jerks) \\
\hline 18 & $\mathrm{C} 5-6$ & ++ & ++ & ++ & - & gait dis. \\
\hline 19 & C5-6 (4-5 (6-7)) & +++ & +++ & +++ & + & (finger jerks) \\
\hline 20 & $\mathrm{C} 4-5$ & + & + & + & - & (unsteadiness) \\
\hline 21 & $C 4-5,5-6$ & +++ & +++ & +++ & + & hyperrefleixa of L/E \\
\hline 22 & $C 4-5,5-6$ & - & - & + & - & \\
\hline 23 & $\mathrm{C} 5-6$ & $++/+++$ & $++/+++$ & $++/+++$ & - & (Wartenberg reflexes) \\
\hline 24 & C6 & - & - & $++/+$ & - & \\
\hline
\end{tabular}

extremities. Thereafter, she developed sensory loss and weakness of both lower extremities. These symptoms moved upward. She complained of a sensation of walking on soft materials. Neurological examination showed no weakness, increased right upper extremity reflexes, normal left upper extremity reflexes with bilateral finger jerks of brachioradial reflexes and finger jerk of left biceps reflex, absence of bilateral lower extremities reflexes, absence of Hoffmann's sign, absence of Babinski sign, absence of sensory level, and absence of sensory disturbance: touch, pinprick, position and vibration senses were intact. MRI demonstrated C4-5 my- elopathy (Fig. 1F, 2G) and L4-5 radiculopathy.

\section{Patient 10}

A 46-year-old man developed gait disturbance, anuresis and impotence. It became difficult for him to go upstairs and downstairs. Neurological examination showed absence of biceps and brachioradial reflexes with bilateral finger jerks of biceps and brachioradial reflexes, normal triceps reflexes, PTRs and ATRs, absence of sensory level, absence of Romberg sign, and normal gait but he was unable to perform tan- 
dem gait. MRI demonstrated C5-6 (and mild C4-5) myelopathy with deformity of the spinal cord (Fig. 1J).

\section{Patient 14}

A 71-year-old woman developed a tingling sensation of the right lower extremity. Neurological examination showed decreased left biceps, bilateral triceps, and right ATR with bilateral finger jerks of biceps and brachioradial reflexes, increased right biceps reflex, and C5-6 sensory level with sacral sparing. MRI demonstrated C5-6 myelopathy.

\section{Patient 24}

A 58-year-old woman developed clumsiness and tingling sensations of both hands five years earlier. Although she underwent surgery on the wrist and right thumb, she lost the ability to grasp objects and she developed a writing disturbance. At 44 years of age, she developed diabetes mellitus. Neurological examination showed atrophy of bilateral abductor policis brevis (APB), weakness of the left ADM, hypotonicity (passivity), generalized areflexia, normal left triceps reflex, decreased right triceps reflex, absence of Hoffmann's sign, absence of Wartenberg (finger flexor) reflex, absence of sensory level, normal touch, pinprick, vibration, and position senses, absence of Romberg sign, and right side dominant decreased arm swing while walking. She was able to perform tandem gait. MRI demonstrated C6 myelopathy (Fig. 1T).

As seen in the lesions in Table 1 and 2, 11 of 24 patients showed bilateral finger jerks associated with C5-6 or C4-5 intervertebral level lesions (C7 or C6 spinal cord lesions). Seven patients showed bilateral finger jerks with hyperreflexia, whereas four patients showed bilateral finger jerks with hyporeflexia or areflexia. Among seven patients who showed bilateral finger jerks and hyperreflexia, two had C4-5 intervertebral level lesions (C6 spinal cord lesions), two had C5-6 intervertebral level lesions (C7 spinal cord lesions), two had both C4-5 and C5-6 intervertebral level lesions (C6 and $\mathrm{C} 7$ spinal cord lesions), and one had both C3-4 and C4-5 intervertebral level lesions (C5 and C6 spinal cord lesions). On the other hand, among four patients who showed bilateral finger jerks with hyporeflexia, two had C5-6 intervertebral level lesions (C7 spinal cord lesions), and two had C4-5 intervertebral level lesions (C6 spinal cord lesions). Nine patients showed generalized hyperreflexia. Five patients showed sensory levels. None of the patients showed Hoffmann's sign. Sixteen patients developed sensory symptoms. At least 16 patients developed symptoms indicating long tract sign. Fourteen patients developed tingling sensations of hands: five patients were unilateral and nine patients were bilateral. Among 14 patients with tingling sensations of hands, seven patients had C4-5 intervertebral level lesions; six patients had C5-6 intervertebral level le- sions; two patients had C6-7 intervertebral level lesions; one patient had C3-4 intervertebral level lesion, and one patient had C6 vertebral level lesion. Nine patients developed motor symptoms. Two patients developed autonomic symptoms. Therefore, 23 of 24 patients (96\%) complained of tingling sensations of hands or showed bilateral finger jerks or generalized hyperreflexia.

\section{Discussion}

Early diagnosis of cervical compressive myelopathy is very important. Sometimes cervical compressive myelopathy patients are misdiagnosed as having peripheral neuropathy, cerebral infarction or other disorders. Some patients were complicated by other disorders. Therefore, accurate diagnoses are necessary in order to relieve patient's symptoms or determine surgical indications. Since patients with intracranial lesions such as stroke may show unilateral finger jerks but do not show bilateral finger jerks, this point is useful for differential diagnosis.

In the present study, complaints of tingling sensations of hands or bilateral finger jerks or generalized hyperreflexia were observed in 23 of 24 cervical myelopathy patients (96\%). All patients with bilateral finger jerks demonstrated C5-6 or C4-5 intervertebral level lesions. Fourteen patients developed tingling sensations of the hands: five patients were unilateral and nine patients were bilateral.

Therefore, these two signs of tendon reflexes seem to be important clues in screening for cervical compressive myelopathy. Previously, bilateral finger jerks with hyperreflexia were interpreted as a sign of hyperreflexia. Therefore, it has meant more rostral lesions in the corticospinal tracts than C6. However, the results of this study suggest that the presence of bilateral finger jerks with hyperreflexia could also be attributed to corticospinal tract lesions owing to C6 (or C7) segmental lesions as well as bilateral finger jerks with areflexia.

Hoffmann's sign and dynamic Hoffmann's test were useful clinical signs for early diagnosis of cervical spondylotic myelopathy $[10,11]$. However, there was no Hoffmann's sign in any of the patients in this study. This may be because the patients in this study had milder myelopathy or they were in an earlier stage than patients in previous studies.

The problem of this study includes as following. Bilateral finger jerks with hyperreflexia or areflexia of biceps and brachioradial reflexes were caused by $\mathrm{C} 5-6$ intervertebral level lesions. Disturbances of refex arcs cause hyporeflexia or areflexia of biceps and brachioradial reflexes. In addition, biceps brachii and brachioradialis are predominantly innervated by C6 (and C5). However, C7 spinal cord corresponds to C5-6 intervertebral disk level. Therefore, if biceps and brachioradial reflexes are $\mathrm{C} 6$ segment monosynaptic reflexes, hyporeflexia or areflexia of biceps and brachioradial 
reflexes owing to $\mathrm{C} 7$ segment compression are difficult to be explained. Possible explanations include as followings. 1) $C 7$ spinal cord segment is involved in reflex arc. 2) Some fibers of reflex arc go down through $\mathrm{C} 7$ spinal cord segment. 3) C5-6 intervertebral level compression affects C6 spinal cord segment. 4) C6 spinal cord segment exists at C5-6 intervertebral disk level. Reflex center of brachioradial reflex may involve C7 spinal cord segment [12]. If brachioradial reflex center involves $\mathrm{C} 7$, hyporeflexia or areflexia of brachioradial reflexes owing to C5-6 intervertebral level compression can be explained. On the other hand, hyperreflexia of biceps and brachioradial reflexes owing to C5-6 intervertebral level lesions are because $\mathrm{C} 7$ spinal cord segment lesion is stimulatory to C6 spinal cord segment.

The authors conclude that deep tendon reflex examination especially observation of bilateral finger jerks or generalized hyperreflexia and history taking of tingling sensations of hands are useful for the screening of cervical compressive myelopathy.

\section{References}

1. Seichi A, Takeshita K, Kawaguchi H, Matsudaira K, Higashikawa A, Ogata N, Nakamura K. Neurologic level diagnosis of cervical stenotic myelopathy. Spine (Phila Pa 1976) 2006;31(12):1338-1343.

2. Matsumoto $M$, Ishikawa $M$, Ishii $K$, Nishizawa $T$, Maruiwa H, Nakamura M, Chiba K, et al. Usefulness of neurological examination for diagnosis of the affected level in patients with cervical compressive myelopathy: prospective comparative study with radiological evaluation. J Neurosurg Spine 2005;2(5):535-539.
3. Yamazaki Y, Tachibana S, Yada K. [Characteristic neurological signs in patients with cervical disc disease]. No Shinkei Geka 1995;23(10):875-880.

4. Adams KK, Jackson CE, Rauch RA, Hart SF, Kleinquenther RS, Barohn RJ. Cervical myelopathy with false localizing sensory levels. Arch Neurol 1996;53(11):11551158.

5. Simmons Z, Biller J, Beck DW, Keyes W. Painless compressive cervical myelopathy with false localizing sensory findings. Spine (Phila Pa 1976) 1986;11(9):869872 .

6. Nakajima M, Hirayama K. Midcervical central cord syndrome: numb and clumsy hands due to midline cervical disc protrusion at the C3-4 intervertebral level. J Neurol Neurosurg Psychiatry 1995;58(5):607-613.

7. Wells P. Cervical angina. Am Fam Physician 1997;55(6):2262-2264.

8. Watson JC, Broaddus WC, Smith MM, Kubal WS. Hyperactive pectoralis reflex as an indicator of upper cervical spinal cord compression. Report of 15 cases. J Neurosurg 1997;86(1):159-161.

9. Montgomery DM, Brower RS. Cervical spondylotic myelopathy. Clinical syndrome and natural history. Orthop Clin North Am 1992;23(3):487-493.

10. Sung RD, Wang JC. Correlation between a positive Hoffmann's reflex and cervical pathology in asymptomatic individuals. Spine (Phila Pa 1976) 2001;26(1):6770 .

11. Denno JJ, Meadows GR. Early diagnosis of cervical spondylotic myelopathy. A useful clinical sign. Spine (Phila Pa 1976) 1991;16(12):1353-1355.

12. Hirayama K. Neurological semiology [in Japanese]. Tokyo: Bunkoudou 1971;518-529. 